\author{
D. Poenaru · D. A. Jacobs · I. Kamal
}

\title{
Unusual findings in the inguinal canal: a report of four cases
}

Accepted: 6 January 1998

\begin{abstract}
Masses in the inguinal canal other than hernias are rare occurrences, and their preoperative diagnosis requires a high index of suspicion. A soft, partly reducible groin mass in a 3-month-old boy proved to be a cystic lymphangioma within the inguinal canal. A $15-$ month-old female who presented with an irreducible inguinal mass was found to have a neuroblastoma metastasis in the groin. An irreducible groin mass in a 6-year-old female proved to be an inguinal canal epidermal inclusion cyst. A 14-yearold female presented with a painful groin swelling that represented an incarcerated hemorrhagic ovarian cyst. An awareness of the wide spectrum of entities other than the standard bowel, testicle, and ovary in the inguinal canal can help to identify uncommon pathologies preoperatively.
\end{abstract}

Key words Inguinal canal - Ovarian cyst · Lymphangioma .

Neuroblastoma $\cdot$ Epidermal cyst

\section{Introduction}

Although the overwhelming majority of masses in the inguinal canal (IC) in

D. Poenaru $(\bowtie) \cdot$ D.A. Jacobs · I. Kamal Department of Surgery, Kingston General Hospital, Queen's University, 76 Stuart Street, Kingston, ON, K7L 2V7, Canada children are inguinal hernias, other rare lesions may occur. We report four cases of children presenting with groin masses that proved to be previously unreported lesions of the IC in the pediatric age group.

\section{Case reports}

Case 1

A 3-month-old boy presented with a soft right groin mass as well as a right scrotal hydrocele. Exploration revealed a cystic mass filled with turbid fluid arising from the right spermatic cord, which did not communicate with the abdominal cavity. Pathology identified the mass as a lymphangioma. The right hydrocele sac was also identified and opened widely. Postoperative abdominal and pelvic ultrasound (US) ruled out any associated lesions. The patient is well with no recurrence at 1-year follow-up.

\section{Case 2}

A 15-month-old female presented with an irreducible, mobile, non-tender, $1.5 \times 1-\mathrm{cm}$ left groin nodule. Groin exploration revealed a subcutaneous mass within the IC, which was excised. Frozen-section examination identified the mass as a neuroblastoma (NB) deposit (Fig. 1). An incidental hernial sac separate from the NB was identified and a hernia repair performed. A subsequent computer tomography (CT) scan revealed the primary tumor, a paraaortic NB, which was subsequently successfully resected. A small labial mass identified on CT was also resected and found to be another NB metastasis. The tumor, a stage IV-S, stroma-poor, NB, was treated with adjuvant chemotherapy. The patient remains disease-free 2 years postoperatively.
Case 3

A 6-year-old female presented with an irreducible, mildly tender groin mass noted for 5 days. A tentative diagnosis of an incarcerated ovary in an inguinal hernia was made and a groin exploration undertaken. A $3 \times 2-\mathrm{cm}$ epidermal inclusion cyst was found inside the inguinal canal and was excised. There was no evidence of a hernia. Recovery was uneventful.

Case 4

A 14-year-old, post-menarcheal female presented complaining of right groin pain. Her past history was unremarkable except for a left inguinal hernia repair and right inguinal exploration at 2 weeks of life. Examination revealed no local mass effect in the right groin, but US showed a mass lesion beneath the abdominal wall in that area. A CT scan revealed a $5 \times 2 \times 2-\mathrm{cm}$ mass distal to the cecal tip and extending into the inguinal region. The patient's pain settled and she was discharged. Six months later, however, she returned with severe right groin pain and a palpable tender mass in the right inguinal area. Groin exploration revealed a large, hemorrhagic ovarian cyst in the right ovary within an intact hernial sac (Fig. 2). Successful ovarian cystectomy and hernia repair were curative.

\section{Discussion}

Despite the overwhelming preponderance of inguinal hernias, several other pathologic entities may be encountered in the IC. These include benign and malignant tumors of the various structures within the IC, lipomas, and hematomas. Our short series expands the spectrum of these unusual lesions of the IC in children. 

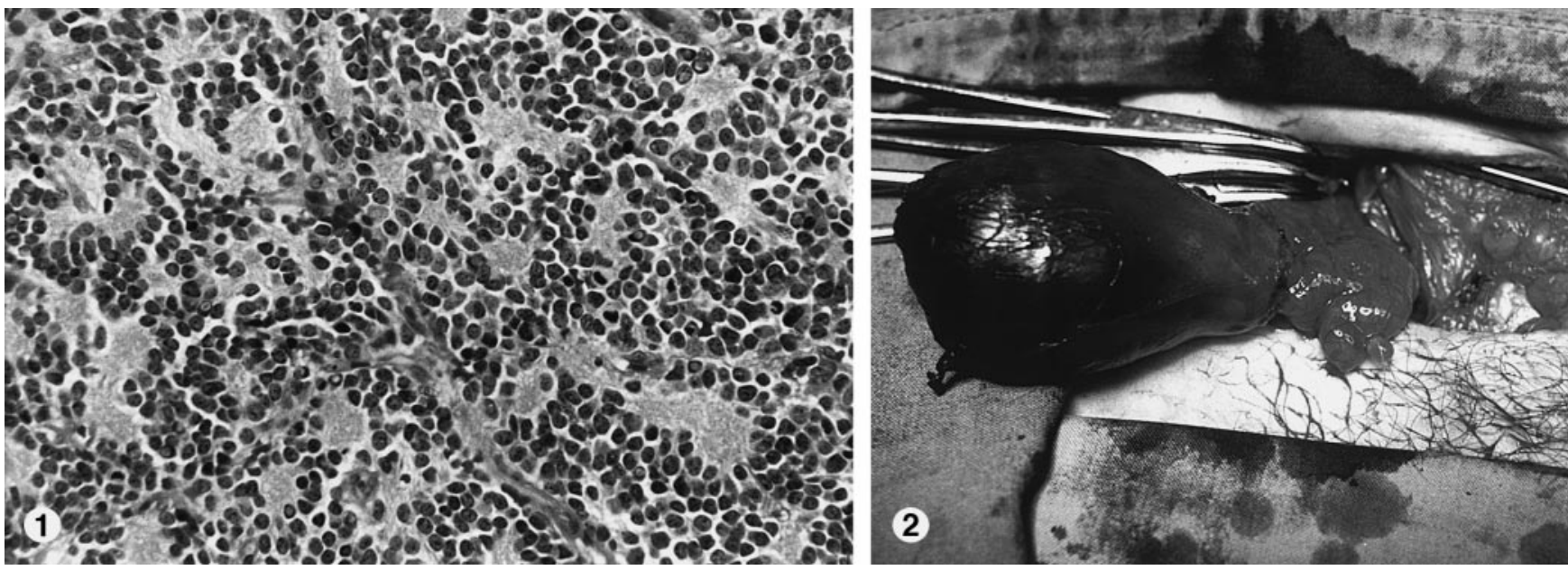

Fig. 1 Histologic slide of groin mass showing neuroblastoma

Fig. 2 Intraoperative photograph of hemorrhagic ovarian cyst within indirect hernia sac

Lymphangiomas can be found anywhere in the body. There have been less than ten cases of intraabdominal lymphangiomas that spread into the IC [5]. One interesting case report describes a lymphangioma of the omentum presenting as an inguinal hernia, and another describes a mediastinal lesion with retroperitoneal extension presenting in the same manner $[1,3]$. Our case is the first instance of an isolated lymphangioma originating in the IC.

NBs of the IC are extremely rare, but metastatic lesions in the pelvis could spread to the canal by pushing through the internal ring. There is one reported case of bilateral primary NBs of the IC [3]. Another case report describes a primary NB of the spermatic cord in a 3-year-old boy [6]. Ours, however, is the first case of a NB metastasis to the IC without associated pelvic pathology.

Dermoid and epidermal cysts are common masses in adults as well as children. Dermoid cysts are malformed epithelial remnants in lines of embryonic fusion, while epidermal inclusion cysts originate from superficial follicles and contain only laminated keratin. Several reports from the adult literature document their rare occurrence in the IC $[2,9]$. The youngest patient previously reported with a dermoid cyst of the IC was 18 years old [7]. The current case adds this pathology to the differential diagnosis of the groin mass in children as well.

Incarcerated adnexa are wellknown in pediatric inguinal hernias in girls, and ovarian cysts have also been reported in this area [4]. The pathology, however, of a hemorrhagic ovarian cyst in an incarcerated inguinal hernia has not been described before. In our patient, the presence of an ovary in the IC went undetected until 14 years of age. It only became evident because the ovary developed a hemorrhagic cyst, which caused painful expansion of the IC.

These cases illustrate that unusual pathology may present in the IC. A reasonable index of suspicion and appropriate investigations will avoid intraoperative surprises as well as benefit the patient by identifying potentially life-threatening lesions.

\section{References}

1. Blumenthal EZ, Gottehrer NP, Dollberg M, et al (1994) A giant tuberculous lymphangioma extending from the mediastinum to the inguinal region. Chest 105: 1279-1280

2. Brightmore T (1971) Dermoid cyst of the inguinal canal simulating a strangulated inguinal hernia. Br J Clin Pract 25: 191

3. Chiari G, Verga G (1989) Cystic lymphangioma of the greater omentum appearing ass an inguinal hernia in an 18-month-old child. Chirurg Pediatr 30: 290-291

4. Goske MJ, Emmens RW, Rabinowitz R (1984) Inguinal ovaries in children demonstrated by high resolution real-time ultrasound. Radiology 151: 635-636

5. Kafka V, Novak K (1970) Multicystic retroperitoneal lymphangioma in an infant appearing as an inguinal hernia. J Pediatr Surg 5: 573

6. Knoedler CJ, Kay R, Knoedler JP Sr, et al (1989) Pelvic neuroblastoma. J Urol 141: 905

7. Leeming R, Olsen M, Ponsky JL (1992) Inguinal dermoid cyst presenting as an incarcerated inguinal hernia. J Pediatr Surg 27: 117-118

8. Leo ME, McManus MJ, Strawbridge LR, et al (1992) Bilateral primary neuroblastoma in the inguinal canal. J Urol 148: 135-137

9. Warren NP, Ralphs DN, Brew DS (1986) A very rare dermoid cyst with a new observation regarding pathogenesis. Br J Clin Pract 40: 31-32 